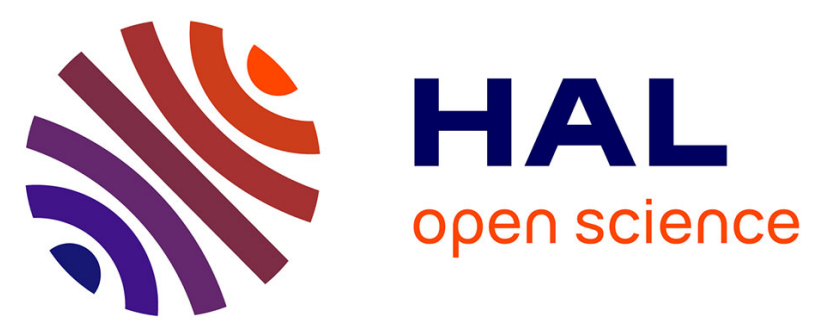

\title{
Salivary adiponectin levels are associated with training intensity but not with bone mass or reproductive function in elite Rhythmic Gymnasts
}

Nikolaos D Roupas, Laurent Maïmoun, Irene Mamali, Olivier Coste, Alexandra Tsouka, Krishna Kunal Mahadea, Thibault Mura, Pascal Philibert, Laura Gaspari, Denis Mariano-Goulart, et al.

\section{To cite this version:}

Nikolaos D Roupas, Laurent Maïmoun, Irene Mamali, Olivier Coste, Alexandra Tsouka, et al.. Salivary adiponectin levels are associated with training intensity but not with bone mass or reproductive function in elite Rhythmic Gymnasts. Peptides, 2014, 51, pp.80 - 85. 10.1016/j.peptides.2013.11.003 . hal-02537291

\author{
HAL Id: hal-02537291 \\ https://hal.umontpellier.fr/hal-02537291
}

Submitted on 10 Apr 2020

HAL is a multi-disciplinary open access archive for the deposit and dissemination of scientific research documents, whether they are published or not. The documents may come from teaching and research institutions in France or abroad, or from public or private research centers.
L'archive ouverte pluridisciplinaire HAL, est destinée au dépôt et à la diffusion de documents scientifiques de niveau recherche, publiés ou non, émanant des établissements d'enseignement et de recherche français ou étrangers, des laboratoires publics ou privés. 


\title{
Salivary adiponectin levels are associated with training intensity but not with bone mass or reproductive function in elite Rhythmic Gymnasts ${ }^{\text {th }}$
}

\author{
Nikolaos D. Roupas ${ }^{a}$, Laurent Maïmoun ${ }^{\mathrm{b}, c, \mathrm{~d}}$, Irene Mamali ${ }^{\mathrm{a}}$, Olivier Coste ${ }^{\mathrm{b}, \mathrm{e}}$, \\ Alexandra Tsouka ${ }^{a}$, Krishna Kunal Mahadea ${ }^{\mathrm{f}}$, Thibault Mura ${ }^{\mathrm{g}, \mathrm{h}}$, Pascal Philibert ${ }^{\mathrm{b}}$, \\ Laura Gaspari $^{\mathrm{b}, \mathrm{e}, \mathrm{i}}$, Denis Mariano-Goulart ${ }^{\mathrm{c}}$, Michel Leglise ${ }^{\mathrm{j}}$, Charles Sultan ${ }^{\mathrm{b}, \mathrm{i}}$, \\ Neoklis A. Georgopoulos $\mathrm{a}, \mathrm{j}$,
}

\author{
a Division of Reproductive Endocrinology, Department of Obstetrics and Gynecology, University of Patras Medical School, University Hospital, Patras, Greece \\ b Département d'Hormonologie, Hôpital Lapeyronie, CHRU Montpellier et UMI, Montpellier, France \\ ' Service de Médecine Nucléaire, Hôpital Lapeyronie, CHRU Montpellier, France \\ d Physiologie et Médecine Expérimentale du Cœur et des Muscles, INSERM U1046, Montpellier, France \\ e Direction Régionale de la Jeunesse, des Sports et de la Cohésion Sociale, Montpellier, France \\ ${ }^{\mathrm{f}}$ Diagnostic Medical Systems (DMS)-Apelem Mauguio, France \\ g Centre d'Investigation Clinique et Département d'Information Médicale, CHRU Montpellier, Montpellier, France \\ h Centre d'Investigation Clinique 1001, INSERM, Montpellier, France \\ i Unité d'Endocrinologie et Gynécologie Pédiatrique, Hôpital A. de Villeneuve, CHRU Montpellier et UMI, Montpellier, France \\ ${ }^{\mathrm{j}}$ Federation Internationale de Gymnastique (FIG) Medical Committee, Lausanne, Switzerland
}

\begin{abstract}
A B S T R A C T
Elite Rhythmic Gymnasts (RGs) constitute a unique metabolic model and they are prone to developing Anorexia Athletica. The aim of the present study was to evaluate the effect of training intensity on salivary adiponectin levels and assess a possible role of salivary adiponectin levels as a predictive factor of reproductive dysfunction and bone mass acquisition in elite RGs. The study included 80 elite female RGs participating in the World Rhythmic Gymnastics Championship tournament held in Montpellier, France on September 2011. Anthropometric values were assessed, training data and menstrual pattern were recorded, bone mass was measured with Broadband ultrasound attenuation (dB/Mhz) and baseline salivary adiponectin levels were determined. The athletes were classified as intensely and very intensely trained, considering the mean training intensity ( $40.84 \mathrm{~h} /$ week). Moreover, considering their reproductive status, they were divided into RG's with normal menstruation, primary amenorrhea and oligomenorrhea. All comparisons were adjusted to age, BMI and body fat percentage differences. Very intensely trained RGs showed higher salivary adiponectin levels $(p=0.05)$. Moreover, salivary adiponectin levels showed significant correlation with training intensity $(r=0.409, p=0.003)$. On the other hand, no association of salivary adiponectin levels was documented with either reproductive function or bone mass acquisition. The results of the present study suggest that, in elite RGs, salivary adiponectin levels are associated with the intensity of training, possibly reflecting the deterioration of energy balance rather than the training stress. On the other hand, a predictive role of salivary adiponectin levels in reproductive dysfunction or bone mass acquisition could not be supported.
\end{abstract}

Adiponectin

Saliva

Gymnasts

Bone mass

Menstrual function

\section{Introduction}

Abbreviations: EDs, eating disorders; AA, anorexia athletica; AN, anorexia nervosa; EDNOS, eating disorders not otherwise specified; RGs, Rhythmic Gymnasts; IR, insulin resistance.

is Grants: This work was supported by grants from Diagnostic Medical Systems (DMS)-Apelem.

* Corresponding author at: Division of Reproductive Endocrinology, Department of Obstetrics and Gynecology, University of Patras Medical School, University Hospital, 26500 Patras, Greece. Tel.: +30 2610999835; fax: +30 2610993854.

E-mail address: neoklisg@hol.gr (N.A. Georgopoulos).
Eating disorders (EDs) encountered in elite athletes have raised great concern and scientific interest and comprise a distinct entity known as anorexia athletica (AA) [24]. Although AA resembles anorexia nervosa (AN), it fails to meet the criteria of EDs and thus it has been suggested to be classified as an eating disorder not otherwise specified (EDNOS) [26].

Elite Rhythmic Gymnasts (RGs) constitute a unique metabolic model prone to develop AA, as the sport specific character favors 
lean physique, leading the athletes to adopt strict dietary restrictions, low caloric intake and chronic negative energy balance (inadequate energy consumption failing to meet energy demands). The inadequate energy consumption characterizing EDs represents a powerful stimulus to the endocrine system, leading to detrimental effects, predominantly involving the reproductive system (primary or secondary amenorrhea, chronic anovulation) and, subsequently, bone metabolism and bone mass (osteopenia and osteoporosis) [22].

During the last decades, the accrual of a large body of evidence has highlighted the emerging role of adipose tissue as an active endocrine organ producing and secreting a number of bioactive molecules (known as adipokines) [6]. Among the numerous adipokines, adiponectin is secreted exclusively from adipose tissue, detected in high levels in serum and involved in metabolism, insulin resistance (IR), inflammatory process and reproductive function [6,12]. Moreover, adiponectin also has an impact on bone metabolism and high adiponectin levels have been shown to increase both osteoclastic and osteoblastic activity [13,14].

Thus, it would be challenging to study the adaptations and variations of adiponectin levels in specific models of energy homeostasis, such as athletes of the highest competitive level. However, a major problem in studying elite athletes on the field of competition lies on the difficulty to obtain blood samples. Thus, the determination of salivary hormone levels provides a convenient, non-invasive and stress-free alternative to blood analysis. In this direction, we [15] and others [25] have reported the determination of adiponectin in saliva and the significant association of salivary with serum adiponectin levels.

Recently, we reported higher levels of salivary adiponectin levels in elite RGs compared to age-matched nonathletic controls [23]. The aim of the present study was to evaluate the effect of training intensity on salivary adiponectin levels, assess a possible role of salivary adiponectin levels as a predictive factor of reproductive dysfunction and bone mass acquisition and further investigate the hypothesis that adiponectin acts as a starvation hormone, signaling low energy availability.

\section{Subjects and methods}

\subsection{Subjects}

The study was conducted during the 2011 World Rhythmic Gymnastics Championship tournament held in Montpellier, France on September 2011 and included 80 elite RGs from 25 countries. The study protocol was approved by the Ethics Review Committee of Nimes, France (Commission de Protection des Personnes, Sud Mediterranee III) and permission for the clinical trials was granted by the French Medicine and Health Care Products Regulatory Agency (Agence Française de Securite Sanitaire des Produits de Santé). Moreover, the study protocol was authorized by the International Gymnastics Federation (FIG).

The athletes participated voluntarily in all parts of the study and informed consent was obtained from athletes, their coaches and parents, in accordance with article 7 of the medical organization of F.I.G. competitions. Before the study (sampling procedure and questionnaire completion), all athletes were familiarized with the sampling techniques and informed about the protocol and the objectives of the study.

\subsection{Methods}

The study protocol included noninvasive clinical and laboratory investigations as well as the completion of a questionnaire. Standing height was measured with a stadiometre to the nearest
$0.1 \mathrm{~cm}$ and recorded as the mean of two consecutive measurements. Body composition and body weight were evaluated using a portable apparatus (Tanita, BC-418MA, TANITA UK LTD), calculating body fat and total body water, via Bioelectrical Impedance Analysis. Body mass index (BMI) was calculated as weight $(\mathrm{kg})$ divided by the square of height $(\mathrm{m})\left(\mathrm{kg} / \mathrm{m}^{2}\right)$. Furthermore, the athletes completed a questionnaire including training history data (age of training onset and usual training intensity).

\subsubsection{Questionnaires}

The participants completed a series of questionnaires designed to assess the general medical and menstrual history, with questions about the age of menarche and the pattern of menses, including the menstrual cycle duration and the absence or irregularity of menstruation. Primary amenorrhea was defined as absence of menstruation above 15 years of age, secondary amenorrhea was defined as absence of menstruation for 3 months in the post-menarche period and in the absence of pregnancy and oligomenorrhea was defined the presence of menstrual interval of more than 35 days, with four to nine periods in the past year. The use of oral contraceptive pills (OCP) was also recorded and OCP users were excluded from the study. The latter might explain the absence of RGs reporting secondary amenorrhea in the studied population. It should also be noted that 3 (three) athletes failed to complete the questionnaire regarding the menstrual status.

In addition, detailed information about the training history was collected, including data on the age of training onset and training intensity (hours per week).

\subsubsection{Quantitative ultrasound measurement}

Quantitative ultrasound (QUS) measurements were made with the Osteospace densitometer (Medilink, Mauguio, France). Osteospace measures broadband ultrasound attenuation (BUA in $\mathrm{dB} / \mathrm{MHz}$ ). The variables are automatically computed after the ultrasonic wave has transversed the calcaneus. The dominant heel was measured in all subjects. The precision (reproducibility) error for BUA is $1.72 \%$ [19].

\subsubsection{Assays}

Saliva samples were collected using Salivettes (Sarstedt Co. Ltd., Nümbrecht, Germany). Saliva samples from athletes were obtained during the tournament in the morning (0800-1000 h) after an overnight fasting, before brushing their teeth.

After centrifugation ( $3000 \mathrm{rpm}$ for $15 \mathrm{~min}$ ) at room temperature, saliva samples were stored at $-20^{\circ} \mathrm{C}$ until the assay. Salivary adiponectin levels were measured using commercially available enzyme immunoassay kits for serum determinations with minor modifications, as we reported in a recent study [15].

Salivary adiponectin concentrations were measured by the quantitative sandwich enzyme immunoassay technique (R\&D Systems, United Kingdom) with dynamic range: $3.9-125.0 \mathrm{ng} / \mathrm{ml}$. The method's sensitivity was $0.246 \mathrm{ng} / \mathrm{ml}$ and the intra- and interassay CVs were $2.5 \%$ and $5.8 \%$, respectively. Salivary adiponectin determinations were carried out at the Laboratory of Reproductive Endocrinology of Patras Medical School.

\subsubsection{Statistical analysis}

All statistical procedures were performed using SPSS 19.0 for Windows (IBM SPSS Statistics, IBM software). Test for normality was done using Kolmogorov-Smirnov test. Variables normally distributed are presented as mean \pm S.D, while skewed variables are presented as median and interquartile range (25th and 75th).

Comparison of mean values between RGs subgroups was performed using the independent t-test or one-way ANOVA when data distribution was normal and Mann-Whitney or Kruskall-Wallis test when the continuous variables were nonnormally distributed. 
Table 1

Anthropometric characteristics, training history and salivary adiponectin levels in elite RGs, considering the training intensity. ${ }^{\text {a }}$

\begin{tabular}{|c|c|c|c|c|}
\hline & Total RGs $(n=80)$ & $\begin{array}{l}\text { RGs with very intense training } \\
(>41 \text { h/week })(n=33)\end{array}$ & $\begin{array}{l}\text { RGs with intense training } \\
(\leq 41 \mathrm{~h} / \text { week })(n=47)\end{array}$ & $p$ value \\
\hline Age (years) & $18.3 \pm 2.6$ & $19.1 \pm 2.99$ & $17.8 \pm 2.3$ & 0.051 \\
\hline Body weight (kg) & $52.0 \pm 4.8$ & $51.5 \pm 5.4$ & $52.6 \pm 4.3$ & 0.331 \\
\hline BMI $\left(\mathrm{kg} / \mathrm{m}^{2}\right)$ & $18.6 \pm 1.3$ & $18.6 \pm 1.5$ & $18.7 \pm 1.1$ & 0.776 \\
\hline Body fat (\%) & $13.5 \pm 3.3$ & $13.8 \pm 3.5$ & $13.2 \pm 3.0$ & 0.498 \\
\hline Onset of training (years of age) & $6.2 \pm 1.7$ & $6.1 \pm 1.6$ & $6.4 \pm 1.9$ & 0.381 \\
\hline Intensity of training (h/week) & $40.8 \pm 13.9$ & $49.4 \pm 2.8$ & $37.1 \pm 6.3$ & 0.000 \\
\hline \multirow{2}{*}{ Salivary adiponectin (ng/ml) } & 2.01 & 2.35 & 1.67 & 0.050 \\
\hline & 1.46 & 1.74 & 1.46 & \\
\hline $\mathrm{BUA}(\mathrm{dB} / \mathrm{Mhz})$ & $67.4 \pm 6.0$ & $67.6 \pm 6.1$ & $67.2 \pm 5.8$ & 0.798 \\
\hline
\end{tabular}

a All variables are presented as mean \pm S.D, while salivary adiponectin levels are presented as median and interquartile range (25th and 75th).

Table 2

Anthropometric characteristics, training history and salivary adiponectin levels in elite RGs, considering the reproductive function.

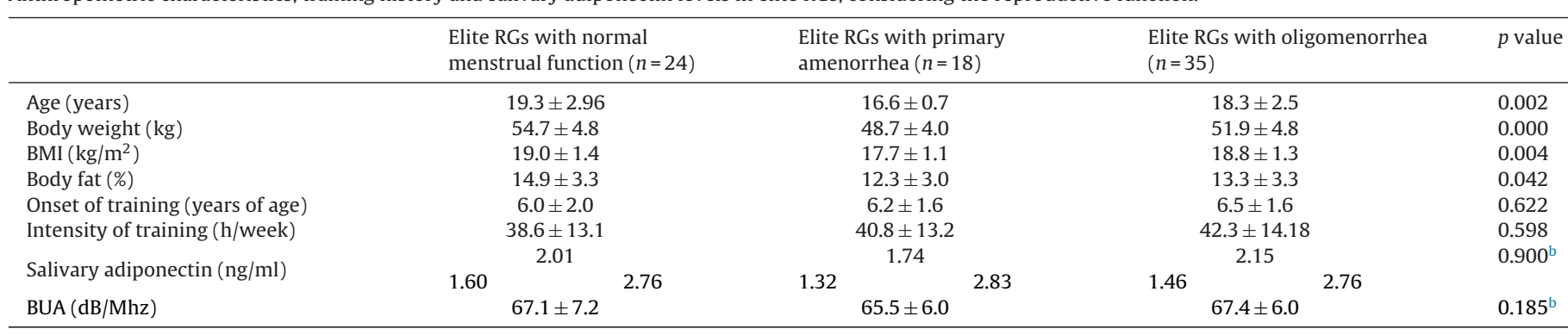

a All variables are presented as mean \pm S.D, while salivary adiponectin levels are presented as median and interquartile range (25th and 75th).

b The comparison has been adjusted to the effect of age and body fat percentage.

The comparison between the sub-groups of reproductive function was adjusted to the effect of age, BMI and body fat (\%) using the general linear model. Moreover, a logistic regression was performed to ascertain the effects of BMI, body fat (\%), salivary adiponectin levels and training intensity on the athletes' reproductive function. Variables that did not follow normal distribution were log-transformed in order to apply logistic regression analysis and general linear model. Two-tailed statistical significance was set at $5 \%$.

Bivariate correlations were assessed with Pearson's correlation coefficient and partial correlation was implemented to control for the effect of additional variables. Salivary adiponectin levels were log-transformed in order to perform Pearson's bivariate correlations. Linear regression analysis was implemented in order to assess the independent predictive role of variables significantly correlated with Pearson's correlation coefficient. Correlations with a critical value of $p<0.05$ were considered significant.

\section{Results}

\subsection{The effect of training intensity on salivary adiponectin levels}

Considering the mean training intensity ( $40.84 \mathrm{~h} /$ week), the athletes were classified as RG's with intense ( $<41 \mathrm{~h} /$ week) and very intense training ( $>41 \mathrm{~h} /$ week). Table 1 summarizes the comparison between RGs with intense and very intense training.

No difference in anthropometric characteristics was observed, while the comparison of salivary adiponectin between the two subgroups higher salivary adiponectin levels in the sub-group of very intense training (Table 1: $p=0.050$ ).

\subsection{The association of reproductive function with salivary adiponectin levels}

The participants were classified as normally menstruating $(n=24)$, athletes with primary amenorrhea $(n=18$, absence of menstruation in girls above 15 years) and athletes with oligomenorrhea
( $n=35$, presence of menstrual interval of more than 35 days, with four to nine periods in the past year). Table 2 summarizes the comparison between the specific sub-groups.

Moreover, multinomial regression analysis was performed to ascertain the effects of BMI, body fat (\%), salivary adiponectin levels and training intensity on the athletes' reproductive function, but the created model was not statistically significant (chi square $=10.926, p=0.206$ ), failing to support a predictive role of salivary adiponectin levels on menstrual status.

\subsection{Correlations}

\subsubsection{Salivary adiponectin levels and training intensity}

Salivary adiponectin levels showed a significant correlation with training intensity (Table 3, Fig. 1: $r=0.409, p=0.003$ ). Moreover, linear regression analysis produced a model predicting salivary adiponectin levels' variation (log transformed value) considering the training intensity (Fig. 1: $r=0.409, F=9.631, p=0.003$ ). Age, BMI and body fat (\%) were excluded from the predictive model, since they did not exert a significant correlation with salivary adiponectin levels (Table 3).

\subsubsection{Salivary adiponectin levels and bone mass (BUA)}

No association of salivary adiponectin levels and bone mass (as expressed by BUA) was documented (Table $3: r=-0.014, p=0.924$ ). The correlation product was not altered even after performing partial correlation analysis, controlling for the effect of age, BMI and body fat $(\%)(r=-0.030, p=0.833)$.

\section{Discussion}

Elite RGs Elite Rhythmic Gymnasts (RGs) constitute a unique metabolic model and they are prone to developing AA, as they begin exercise and training at an early age and undergo intensive training programs, resulting in great physical and psychological stress. Furthermore, the sport specific character favors lean physique, leading 
Table 3

Pearson's correlation coefficients between salivary adiponectin levels, anthropometric characteristics, training intensity parameters and BUA (salivary adiponectin levels have been log transformed).

\begin{tabular}{llllll}
\hline & Age & BMI & Body fat (\%) & Age of training onset & Intensity of training \\
\hline Log(adiponectin) & $r=0.177$ & $r=-0.015$ & $r=-0.044$ & $r=-0.24$ & $r=0.409$ \\
& $p=0.219$ & $p=0.918$ & $p=0.760$ & $p=0.873$ & $p=0.003$ \\
\hline
\end{tabular}

the athletes to adopt strict dietary restrictions, low caloric intake and chronic negative energy balance.

The present study reports on the association between salivary adiponectin levels, training intensity, reproductive function and bone mass in elite RG's. The results of the present study document an association of salivary adiponectin levels with training intensity, but no relationship with either menstrual pattern or bone mass acquisition.

\subsection{Salivary adiponectin levels and training intensity}

The results of the present study highlight an association of salivary adiponectin with training intensity (as expressed by the hours of training/week). More specifically, RG's with very intense training (training $\geq 41 \mathrm{~h} /$ week) showed higher salivary adiponectin levels compared to elite RG's of comparable agonistic level adopting more moderate training regimes (training $\leq 41 \mathrm{~h} /$ week).

Up to date, few studies have reported on the effect of exercise on adiponectin levels, concluding that chronic exercise increases adiponectin levels, only when the training program is extended and accompanied by weight loss and body fat reduction [4]. Interestingly, increased adiponectin levels have been reported in female ballet dancers [5], which resemble to a certain degree RG, while studies of prepubertal RG's performing at low competitive level reported no differences in adiponectin levels compared to sedentary controls, matched for age and BMI [20].

Numerous studies, both clinical and experimental, have highlighted a possible role of adiponectin in the regulation of energy homeostasis, leading to rather controversial conclusions [1]. On one hand, it has been postulated that adiponectin increases energy expenditure and fatty acid oxidation and reduces body weight [9], while other studies suggested that adiponectin reduces energy expenditure and increases food intake and body weight [10]. In addition, studies on adolescent females diagnosed with anorexia nervosa reported higher levels of adiponectin and a reversal with weight rehabilitation [18]. These conflicting findings could be attributed to the suggested opposing metabolic roles of adiponectin receptors (AdipoR1 and AdipoR2) [3].

With regards to the role of adiponectin in signaling energy homeostasis in sports and exercise, Jurimae et al. concluded that basal adiponectin levels are not a good marker of energy homeostasis in athletes [8]. This conclusion was drawn from studies regarding elite rowers during prolonged training periods at relatively high daily energy expenditures [8]. Still, these results refer to power athletes, with great muscle mass and high daily energy consumption (in order to reach the high energy expenditure), in contrast to the elite RG's enrolled in the present study, that adopt chronic negative energy balance in order to retain a lean physique.

In elite RGs as the level of competition becomes higher and the expectations for improved performance and international records become greater, the training programs are intensified and the demands to retain lean somatotype deteriorate the energy balance (inadequate energy consumption failing to meet energy demands). Considering that RGs training programs do not involve aerobic exercise or resistance training but high quality technical performance, it could be suggested that the higher adiponectin levels found in highly trained athletes reflect the deterioration of energy balance. Thus, the results of the present study, combined with the

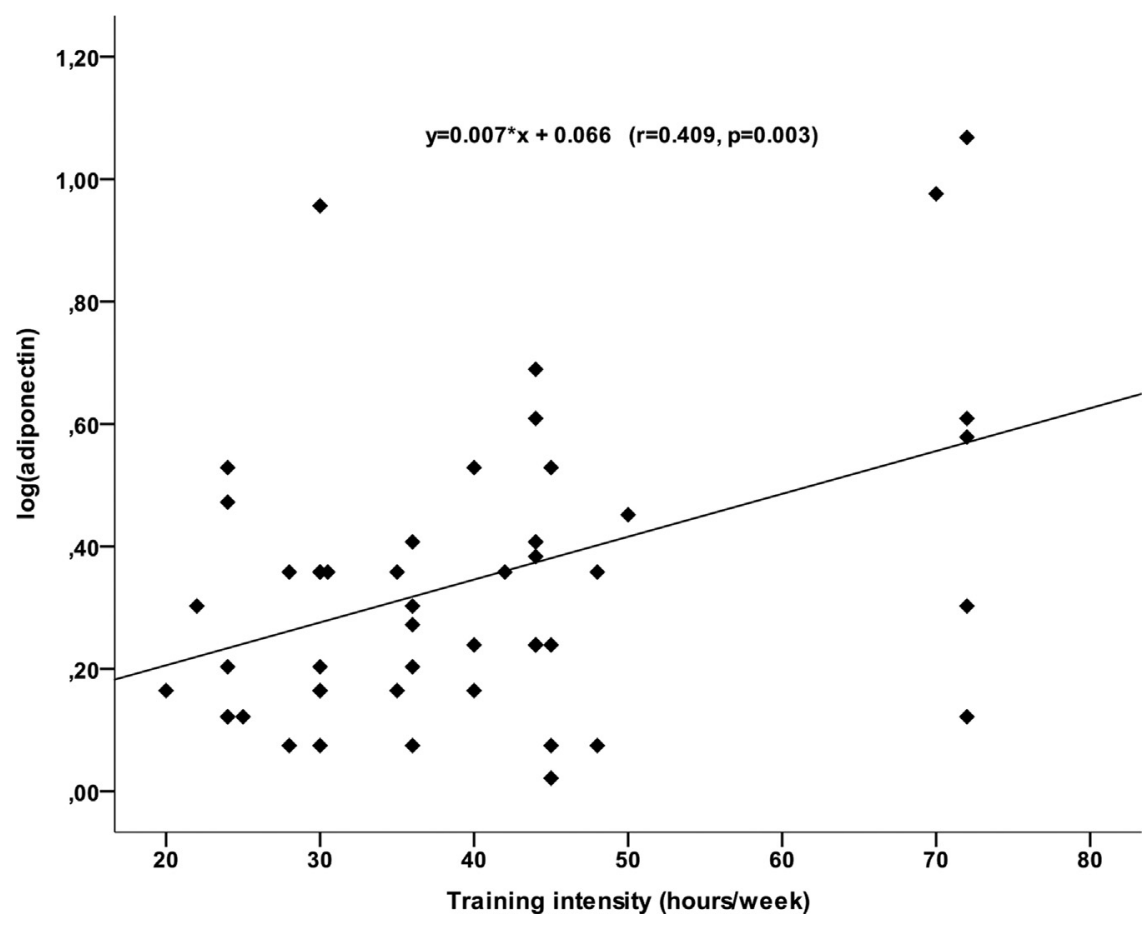

Fig. 1. Salivary adiponectin levels are associated with training intensity in elite RGs. 
aforementioned data and the results of our recently reported study on elite RG's [23], further support the suggested role of adiponectin as a "starvation" hormone, signaling low energy availability [1].

\subsection{Salivary adiponectin levels and reproductive function}

The present study reports no difference in salivary adiponectin levels among normally menstruating, amenorrheic and athletes with oligomenorrhea. This finding should be interpreted under the light of the currently available data that suggest a potential role for adiponectin in the regulation of neuroendocrine reproductive processes [16].

More specifically, it has been reported that adiponectin inhibits both basal and GnRH-stimulated LH secretion in short-term treated rat pituitary cells [21]. Furthermore, Lu et al. reported that adiponectin acutely reduced basal and GnRH-stimulated $\mathrm{LH}$ secretion but had no impact on FSH levels [12]. Thus, it could be hypothesized that increased adiponectin levels (as found in lean and energy restrained female athletes) may contribute to suppression of LH levels and chronic anovulation.

However, the present study failed to document a relationship between salivary adiponectin levels and reproductive status. This discrepancy could be attributed to the relatively small sample size of each sub-group or to the existence of a threshold of adiponectin levels required for the inhibitory effect on hypothalamus to be exerted. Apparently, further research is needed to evaluate a possible role of salivary adiponectin as a marker of reproductive dysfunction in chronic undernutrition states.

\subsection{Salivary adiponectin levels and bone mass}

The present study reports no association of salivary adiponectin levels and bone mass acquisition in elite RGs. Furthermore, no such an association was documented even if the analysis considered either the training intensity (separate analysis within athletes with intense and very intense training) or the reproductive function (separate analysis within normally menstruating, amenorrheic and athletes with oligomenorrhea).

With regards to the association of adiponectin with bone metabolism and bone mass acquisition, it has been shown that high adiponectin levels increase both osteoclastic and osteoblastic activity $[13,14]$. Additionally, inverse associations of adiponectin with bone density are reported in adults [11], while adiponectin has been introduced as an independent predictor of BMD in healthy females [7].

In the case of EDs, although Misra et al. demonstrated that adiponectin was a significant and independent contributor of areal BMD values in healthy adolescent girls and girls with AN [17], the role of adiponectin in bone mass acquisition is still unclear. Moreover, Parm et al, in their study of prepubertal RGs performing at low competitive level, documented no association of plasma adiponectin levels with bone density parameters [20]. Taken together, the results of the present study and the aforementioned data cannot support a predictive role of adiponectin levels in the evaluation of bone mass acquisition in energy deprivation states.

The present study has certain limitations. First of all, this is a field study and carries all the disadvantages of such studies, with regards to sample size, sampling collection and the accuracy of the reported data. Most importantly, the results of the present study refer to measurements of salivary adiponectin levels and the evaluation, generalization and comparison with reported data on blood analysis should be made with great cautiousness. Since no data on salivary adiponectin were available, data from studies implementing blood analysis were used in order to elucidate adiponectin's physiologic response to exercise and energy imbalance and set the background for the interpretation of the reported results. Moreover, saliva sampling and analysis have certain limitations per se: although adiponectin levels were determined in saliva, their salivary levels exerted a weak, though statistically significant, correlation with serum adiponectin levels and are detected in significantly lower levels compared to their relative serum levels $[15,25]$. In addition, salivary hormonal determinations (especially of peptides) are very sensitive and heavily-dependent on certain conditions (sampling technique, storage conditions and laboratory analysis, e.g. saliva sample dilution [2]). Therefore, in the absence of specific and standardized analytical tools (e.g. laboratory techniques designed exclusively for the process of saliva samples), the clinical and diagnostic value of salivary analysis should be limited to the evaluation of tendencies and robust changes, supplementary to blood analysis.

\section{Conclusion}

In elite RGs salivary adiponectin is associated with exercise intensity and may reflect the deterioration of energy balance rather than the exercise stress per se, suggesting a possible role as a marker of energy deprivation. On the other hand, no association of salivary adiponectin levels was documented either with reproductive function or bone mass acquisition.

\section{Disclosure}

The authors declare that they have no conflict of interest.

\section{References}

[1] Ahima RS, Lazar MA. Adipokines and the peripheral and neural control of energy balance. Mol Endocrinol 2008;22:1023-31.

[2] Akuailou E-N, Vijayagopal P, Imrhan V, Prasad C. Measurement and validation of the nature of salivary adiponectin. Acta Diabetol 2013;50(5):727-30.

[3] Bjursell M, Ahnmark A, Bohlooly-Y M, William-Olsson L, Rhedin M, Peng XR, et al. Opposing effects of adiponectin receptors 1 and 2 on energy metabolism Diabetes 2007;56:583-93.

[4] Bouassida A, Chamari K, Zaouali M, Feki Y, Zbidi A, Tabka Z. Review on leptin and adiponectin responses and adaptations to acute and chronic exercise. $\mathrm{Br}$ Sports Med 2010:44:620-30.

[5] Donoso MA, Muñoz-Calvo MT, Barrios V, Garrido G, Hawkins F, Argente J. Increased circulating adiponectin levels and decreased leptin/soluble leptin receptor ratio throughout puberty in female ballet dancers: Association with body composition and the delay in puberty. Eur J Endocrinol 2010;162:905-11.

[6] Galic S, Oakhilla JS, Steinberg GR. Adipose tissue as an endocrine organ. Mol Cell Endocrinol 2010;316:129-39.

[7] Jurimae J, Rembel K, Jurimae T, Rehand M. Adiponectin is associated with bone mineral density in perimenopausal women. Horm Metab Res 2005;37:297-302.

[8] Jürimäe J, Mäestu J, Jürimäe T, Mangus B, von Duvillard SP. Peripheral signals of energy homeostasis as possible markers of training stress in athletes: a review. Metabolism 2011;60:335-50

[9] Kim JY, van de Wall E, Laplante M, Azzara A, Trujillo ME, Hofmann SM, et al. Obesity-associated improvements in metabolic profile through expansion of adipose tissue. J Clin Invest 2007:117:2621-37.

[10] Kubota N, Yano W, Kubota T, Yamauchi T, Itoh S, Kumagai H, et al. Adiponectin stimulates AMP-activated protein kinase in the hypothalamus and increases food intake. Cell Metab 2007;6:55-68.

[11] Lenchik L, Register TC, Hsu FC, Lohman K, Nicklas BJ, Freedman BI, et al. Adiponectin as a novel determinant of bone mineral density and visceral fat. Bone 2003;33:646-51.

[12] Lu M, Tang Q, Olefsky JM, Mellon PL, Webster NJ. Adiponectin activates adenosine monophosphate activated protein kinase and decreases luteinizing hormone secretion in LbetaT2 gonadotropes. Mol Endocrinol 2008:22:760-71.

[13] Luo XH, Guo LJ, Yuan LQ, Xie H, Zhou HD, Wu XP, et al. Adiponectin stimulates human osteoblasts proliferation and differentiation via the MAPK signaling pathway. Exp Cell Res 2005;309:99-109.

[14] Luo XH, Guo LJ, Xie H, Yuan LQ, Wu XP, Zhou HD, et al. Adiponectin stimulates RANKL and inhibits OPG expression in human osteoblasts through the MAPK signaling pathway. J Bone Miner Res 2006;21:1648-56.

[15] Mamali I, Roupas ND, Armeni AK, Theodoropoulou A, Markou KB, Georgopoulos NA. Measurement of salivary resistin, visfatin and adiponectin levels. Peptides 2012;33:120-4.

[16] Michalakis KG, Segars JH. The role of adiponectin in reproduction: from polycystic ovary syndrome to assisted reproduction. Fertil Steril 2010;94:1949-57. 
[17] Misra M, Miller KK, Cord J, Prabhakaran R, Herzog DB, Goldstein M, et al. Relationships between serum adipokines, insulin levels, and bone density in girls with anorexia nervosa. J Clin Endocrinol Metab 2007;92:2046-52.

[18] Modan-Moses D, Stein D, Pariente C, Yaroslavsky A, Ram A, Faigin M, et al. Modulation of adiponectin and leptin during refeeding of female anorexia nervosa patients. J Clin Endocrinol Metab 2007;92(5):1843-7.

[19] Njeh CF, Chen MB, Fan B, Grigorian M, Shepherd JA, Saeed I, et al. Evaluation of a gel-coupled quantitative ultrasound device for bone status assessment. J Ultrasound Med 2001;20:1219-28.

[20] Parm AL, Jürimäe J, Saar M, Pärna K, Tillmann V, Maasalu K, et al. Plasma adipocytokine and ghrelin levels in relation to bone mineral density in prepubertal rhythmic gymnasts. J Bone Min Metab 2011;29:717-24.

[21] Rodriguez-Pacheco F, Martinez-Fuentes AJ, Tovar S, Pinilla L, Tena-Sempere M, Dieguez C, et al. Regulation of pituitary cell function by adiponectin. Endocrinology 2007;148:401-10.
[22] Roupas ND, Georgopoulos NA. Menstrual function in sports, Hormones 2011;10:104-16

[23] Roupas ND, Mamali I, Armeni AK, Markantes GK, Theodoropoulou A Alexandrides TK, et al. The influence of intensive physical training on salivary adipokine levels in elite Rhythmic Gymnasts. Horm Metab Res 2012;44(13):980-6.

[24] Sudi K, Öttl K, Payerl D, Baumgartl P, Tauschmann K, Müller W. Anorexia athletica. Nutrition 2004;20:657-61

[25] Toda M, Tsukinoki R, Morimoto K. Measurement of salivary adiponectin levels. Acta Diabetol 2007;44:20-2.

[26] Warren MP. Endocrine manifestations of eating disorders. J Clin Endocrinol Metab 2011;96:333-43 